\title{
Scaling of turbulence spectra measured in strong shear flow near the Earth's surface
}

Mikkelsen, Torben Krogh; Larsen, Søren Ejling; Ejsing Jørgensen, Hans; Astrup, Poul; Larsén, Xiaoli Guo

Published in:

Physica Scripta

Link to article, DOI:

10.1088/1402-4896/aa91b2

Publication date:

2017

Document Version

Peer reviewed version

Link back to DTU Orbit

Citation $(A P A)$ :

Mikkelsen, T. K., Larsen, S. E., Ejsing Jørgensen, H., Astrup, P., \& Larsén, X. G. (2017). Scaling of turbulence spectra measured in strong shear flow near the Earth's surface. Physica Scripta, 92.

https://doi.org/10.1088/1402-4896/aa91b2

\section{General rights}

Copyright and moral rights for the publications made accessible in the public portal are retained by the authors and/or other copyright owners and it is a condition of accessing publications that users recognise and abide by the legal requirements associated with these rights.

- Users may download and print one copy of any publication from the public portal for the purpose of private study or research.

- You may not further distribute the material or use it for any profit-making activity or commercial gain

- You may freely distribute the URL identifying the publication in the public portal 


\title{
Scaling of Turbulence Spectra Measured in Strong Shear Flow near the Earth's Surface
}

Mikkelsen, T.1 ; S.E. Larsen ${ }^{1}$; H.E. Jørgensen ${ }^{1}$; P. Astrup ${ }^{1}$ and X.G. Larsén ${ }^{1}$

1) DTU Wind Energy, Denmark

\begin{abstract}
Within the lowest kilometer of the Earth's atmosphere, in the so-called atmospheric boundary layer, winds are often gusty and turbulent. Nearest to the ground, the turbulence is predominately generated by mechanical wall-bounded wind shear, whereas at higher altitudes turbulent mixing of heat and moisture also play a role.
\end{abstract}

The variance (square of the standard deviation) of the fluctuation around the mean wind speed is a measure of the kinetic energy content of the turbulence. This kinetic energy can be resolved into the spectral distributions, or spectra, as functions of eddy size, wavenumber, or frequency. Spectra are derived from Fourier transforms of wind records as functions of space or time corresponding to wavenumber and frequency spectra, respectively.

Atmospheric spectra often exhibit different subranges that can be distinguished and scaled by the physical parameters responsible for: 1 ) their generation; 2 ) the cascade of energy across the spectrum from largeto small-scale; and 3) the eventual decay of turbulence into heat owing to viscosity effects on the Kolmogorov microscale, in which the eddy size is only a fraction of a millimeter.

This paper addresses atmospheric turbulence spectra in the lowest part of the atmospheric boundary layer-the so-called surface layer-where the wind shear is strong owing to the nonslip condition at the ground.

Theoretical results dating back to C.M Tchen's early work in 1953 "On the spectrum of energy in turbulent shear flow" led Tchen to predict a shear production subrange with a distinct inverse-linear power law for turbulence in a strongly sheared high-Reynolds number wall-bounded flow, as is encountered in the lowest sheared part of the atmospheric boundary layer, also known as the eddy surface layer.

This paper present observations of spectra measured in a meteorological mast at $H \varnothing v s \varnothing r e$, Denmark that support Tchen's prediction of a shear production subrange following a distinct power law of degree -1 in the lowest part of the atmospheric surface layer with the form $\sim u_{*}^{2} k^{-1}$, where $u_{*}$ is the surface friction velocity and $k$ is the wavenumber. Tchen's turbulence theory is shown to be able to predict the measured spectra of the wind velocity component parallel to the mean wind direction for eddy sizes larger than the measurement height above the ground.

An amended analytical model for the near-neutral surface layer spectrum is then proposed. This model, which is applicable to the scaling of the $u$ spectrum at all heights in the surface layer, is obtained by a combination of J.C. Kaimal's classical spectral model for scaling the inertial subrange with Tchen's 1953 and 1954 proposed shear production subrange theory. 
The shear production-amended spectral model is compared with observations of ensemble-averaged nearneutral spectra selected during a nine-month measurement period from recordings from six sonic anemometers at heights of $10,20,40,60,80$, and $100 \mathrm{~m}$ in the meteorological tower at the test site for large wind turbines in $\mathrm{H} \varnothing \mathrm{v} \varnothing \mathrm{re}$, Denmark.

Finally, potential applications of the new spectral model are discussed, in particular for use within the lowest one-third of the surface layer in which the production subrange component of the spectrum is most prominent. The new spectral model can supply wavenumber-resolved turbulent kinetic energies for the prediction of wind loads on buildings, bridges, and wind turbines, and its spectral parameterization can also be used for scale-dependent parameterization of, e.g., surface-released atmospheric dispersion calculations for regions close to the ground.

\section{Introduction}

\subsection{Motivation and experimental Setup}

In the part of the Earth's atmosphere closest to the ground, where most human activity unfolds, the atmospheric turbulence is strongly influenced by the fluid dynamical constraint referred to as the nonslip boundary condition on the ground, meaning that the wind speed on the Earth's surface is zero; consequently turbulence close to the Earth's surface is often produced and evolving in a strong vertical wind shear.

Measurement and detailed analysis of turbulence in the surface layer of the atmospheric boundary layer, i.e., the layer of the atmosphere directly affected by the structure of the Earth's surface, is important for the prediction and modeling of turbulence and its spectra. Spectra provide important information on the distributions of turbulent kinetic energy broken down according to eddy size or the wavenumber needed to estimate, e.g., wind loads on buildings or forcing of resonance frequencies on bridges and wind turbines, and also to understand and model atmospheric flow and dispersion of pollutants.

This work presents measurements and spectral models of turbulence energy spectra affected by the strong local wind shear near the ground. Figure 1 illustrates our experimental setup for producing the detailed measurements of turbulence used for subsequent spectral analysis and modeling. 


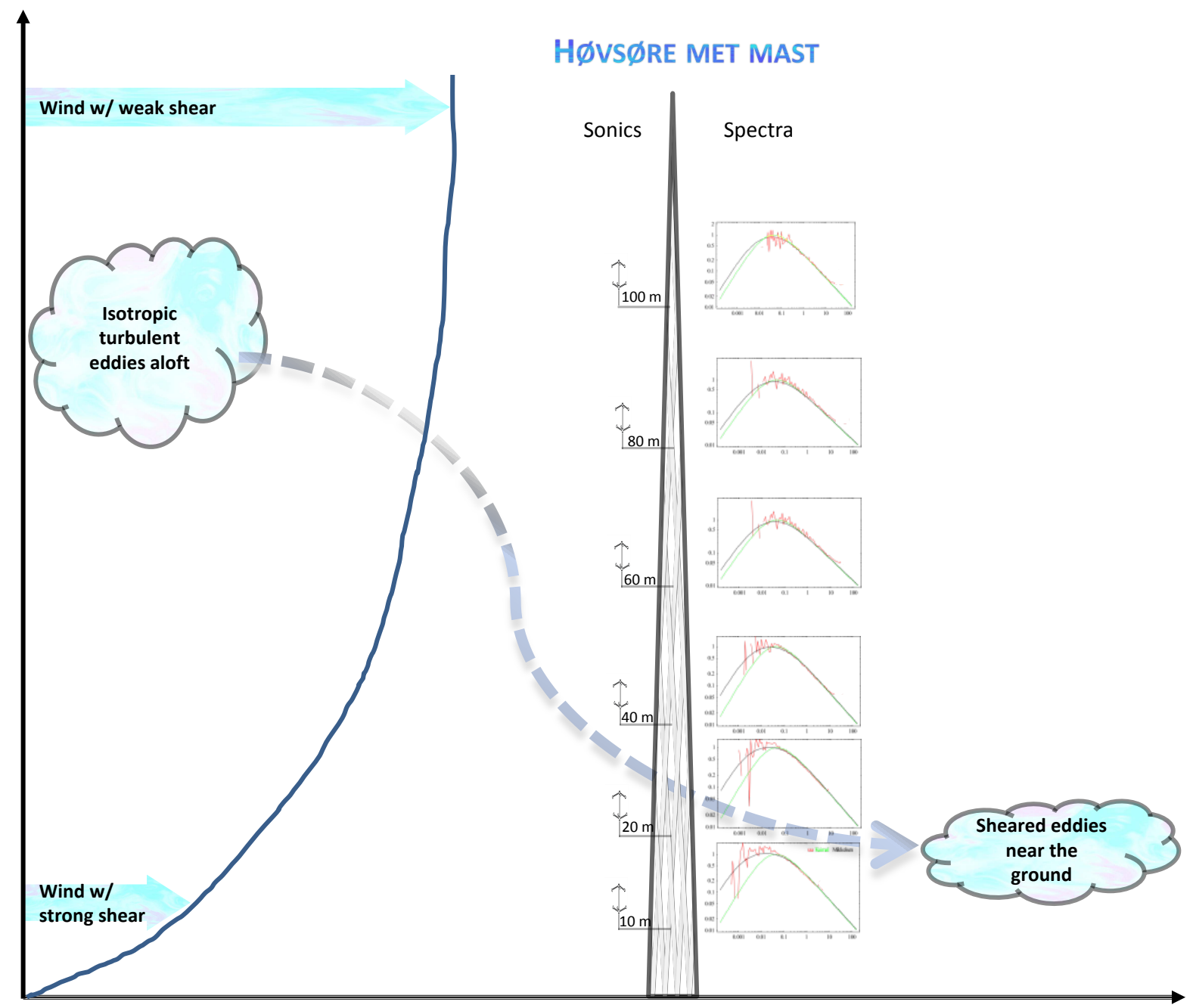

Figure 1. Full-scale field experimental setup located in the 125-m tall meteorological mast at Høvsøre, Jutland, Denmark.

The sonic anemometers installed on the left side of the meteorological tower measure the wind fluctuations at six heights from 10 to $100 \mathrm{~m}$ above the ground. The sonic anemometers measure the time series of the turbulence from which the corresponding energy spectra at each height are calculated, as illustrated by the spectra shown directly to the right of the met tower in the figure.

The $\mathrm{H} \varnothing \mathrm{v} \varnothing \varnothing \mathrm{re}$ met mast is located in a rural area and is exposed to turbulent wind profiles that are strongly sheared, i.e., the wind speed increases strongly with height; the rate of increase is highest closest to the ground and progressively decreases toward the top of the met mast. 
As the three-dimensional structure of the turbulence aloft is less affected by the presence of the ground, the turbulence near the top of the met mast is nearly isotropic, having a common structure in all directions; however, when eddies carrying momentum aloft are advected (transported) down to the ground by downdrafts, the eddies become sheared and elongated, as illustrated by the sweeping of the eddy in Figure 1 through the met mast and toward the ground.

The paper is organized as follows:

In Section 1.2, the general structures of the atmospheric boundary layer flow and turbulence are briefly described, and the present investigation related to the measured $\mathrm{H} \emptyset \mathrm{v} \varnothing \mathbf{r e}$ surface layer spectra is set into an overall atmospheric turbulence spectra context.

Section 2 introduces shear production and inertial spectral subranges.

Section 3 introduces a new combined spectral model of near-neutral surface layer spectra.

In Section 4, the measurements of turbulence spectra in previous investigations and the new spectra from $\mathrm{H} \varnothing \mathrm{v} \varnothing \mathrm{re}$ are analyzed and compared with the amended spectral model developed in Section 3 combining the spectral scaling of C.M Tchen's shear production subrange with the previous suggested Kaimal spectral model for the classical Kolmogorov inertial subrange scaling.

\subsection{The general structure of the atmospheric boundary layer wind speed spectra}

The processes behind atmospheric motion can be considered in terms of relevant characteristic space and time scales. The wind speed spectra of turbulence are derived from Fourier transforms of wind records as functions of space or time corresponding to wavenumber and frequency spectra, respectively. These spectra represent the kinetic energy or variances within different spectral ranges that appear at different frequency and wavenumber regions.

The variance in wind speed, or square of the standard deviation of its fluctuation, $1 / 2 \sigma_{u}^{2}=\int_{0}^{\infty} F_{11}\left(k_{1}\right) d k_{1}$, represents the total kinetic energy contained in the turbulent part of the wind velocity component, $u$, aligned parallel to the mean wind direction. $F_{11}\left(k_{1}\right)$ is the corresponding wavenumber spectrum $\left[\mathrm{m}^{3} \mathrm{~s}^{-2}\right]$; its form reveals how the turbulent kinetic energy is distributed as a function of the eddy size, $\lambda$, which is related to the corresponding wavenumber, $k_{1}=2 \pi / \lambda$.

A stationary sensor will, for broad spectral ranges, see a frequency content dominated by advection by the mean wind speed of the spatially distributed eddy structures. The wavenumber spectrum can also be sampled by remote sensing-based light detection and ranging instruments (lidar) able to scan the turbulent eddy structures or from sensors mounted on an airplane. In this paper, we will consider spectra measured by fast-responding sonic anemometers mounted in-situ at various heights in a $125 \mathrm{~m}$ tall meteorological mast. 
Figure 2 shows the wind speed spectra $n S(n)$ calculated from time series sampled continuously during 2012 and 2013 using sonic anemometers. The spectra show the contribution of the total speed variance at various frequencies, $n(\mathrm{~Hz})$, at three heights $-10,80$, and $100 \mathrm{~m}$-in the meteorological mast installed in 2004 at the test station for large wind turbines in $\mathrm{H} \varnothing \mathrm{v} \varnothing \varnothing \mathrm{re}$, Denmark, which is located approximately $1.7 \mathrm{~km}$ inland from the west coast of Jutland, Denmark [1].
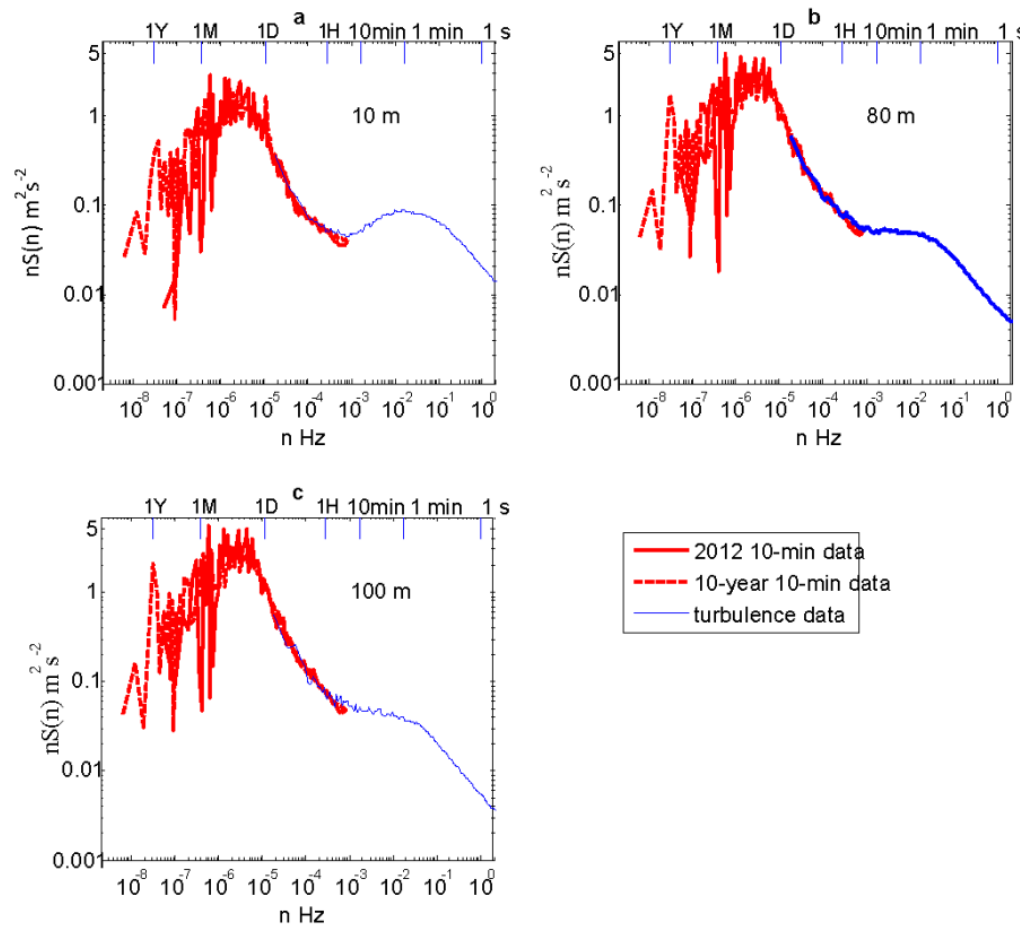

Figure 2. Høvsøre wind speed spectra as a function of frequency [Hz] at heights 10,80 , and $100 \mathrm{~m}[3]$, with associated time scale indicated.

The spectra contain the full range of yearly encountered atmospheric stabilities and are calculated from time series sampled at $20 \mathrm{~Hz}$ using METEK USA 1 three-dimensional sonic anemometer/thermometers.

The spectra show peaks at annual and diurnal frequencies and at a height of $10 \mathrm{~m}$. At frequencies of up to around $\sim 1$ day $^{-1}$, the spectra reflect weather; at higher frequencies, the spectra can be considered to reflect turbulence eddies of various scales, and models have been constructed to parameterize these parts of the spectrum. For frequencies from about $\sim 1$ day $^{-1}$ to $\sim 10^{-4} \mathrm{~Hz}$, the spectrum is denoted "mesoscale" and has a largely two-dimensional turbulence, meaning that it contains much smaller vertical velocity components than horizontal wind components. Additionally, beyond the first 10-50 m above the ground the spectra are found to vary very little with height. In the turbulent parts of the spectra at frequencies above $\sim 1 \mathrm{~min}^{-1}$, the normalized spectra, $n S(n)$, essentially vary as $n^{-2 / 3}$, meaning that the spectrum itself, $S(n)$, diminishes with increasing frequency following a $-5 / 3^{\text {rd }}$-power law. Frequencies of about $10^{-4}$ $\mathrm{Hz}$ and higher are associated with boundary-layer spectra generated by processes within the atmospheric 
boundary layer-approximately the first kilometer above the surface of the Earth, in which atmospheric conditions are strongly influenced by the proximity of the surface. The frequency region between $10^{-4}$ and $10^{-3} \mathrm{~Hz}$ involves a "spectral gap," which is especially evident at lower altitudes (cf. Figure 2) because the spectral energy in the mesoscale spectrum is here quite low and the boundary layer turbulence is weak in this region. At higher frequencies, the boundary layer turbulence is more intense.

For frequencies larger than $\sim 1\left[\right.$ day $\left.^{-1}\right]$, the space-time conversion relation of the spectra can be approximated using Taylor's frozen turbulence hypothesis, meaning that the time variation observed can be explained as essentially being a "frozen" spatial variation that is advected as a solid body by the mean wind speed. This construction becomes increasingly accurate with increasing frequency, and all frequency spectra for boundary layer turbulence are usually interpreted using this hypothesis. Turbulence spectra theory and models are typically formulated in terms of wavelengths or wavenumbers. For measurements using a stationary sensor, the frequencies can be related via the frozen turbulence hypothesis to the relevant wavelengths through $n=u / \lambda$, where $u$ is the mean speed and $\lambda$ is the wavelength corresponding to the wavenumber component $k_{1}=2 \pi / \lambda$ parallel with the mean wind direction. In this manner, one can easily change between wavenumber and frequency spectra.

The surface layer usually refers to the lowest one-tenth of the boundary layer, in which the vertical fluxes of heat, moisture, and momentum are, to a first-order approximation, constant with height. Furthermore, in the lowest one-third of the surface layer, the so-called "eddy-surface layer" (cf. [2] and [3]), production of turbulence is dominated by impinging eddies from above that are stretched out as they approach the surface and replicate themselves in intense local shear near the surface (cf. the generation of "cat paws" that can be observed when strong downdrafts impinge onto calm sea surfaces); these generate a spectrum of spectral form $k^{-1}$, which we shall refer to the "shear production subrange" (cf. [4]), a term first introduced by Tchen $[5,6]$. The spectra in Figure 2 represent ten-year averaged wind speed spectra at three heights that contain all encountered stability ranges, including convective conditions, which also generate turbulence in the shear production spectral range. However, as we shall see the shear production subrange of interest in this study is most profoundly observed during near-neutral atmospheric stability conditions and at heights close to the ground. In Fig 2a, the shear production subrange is embedded in the segment of the spectral peak corresponding to a frequency range between $10^{-1}$ and $10^{-3} \mathrm{~Hz}$; however, in this range the year-averaged spectra shown in Figure 2 are strongly influenced by non-neutral, in-particular convective(upward heat flux) generated turbulence. Consequently, to eliminate the dominance of convective as well as stable (downward heat flux) stratification-generated spectra from the shear-produced spectra in question, only spectra that have been observed during near-neutral atmospheric stability conditions are selected for the present analysis, cf. Section 3.

The smallest eddy sizes to be contained within the shear production subrange are of size $\lambda \approx z$, where $z$ is the measurement height above the ground. Eddies of this size and larger are not isotropic; rather, they "sense" the presence of the ground below. On the other end of the scale, the largest eddy sizes to be found within the shear production subrange are of size comparable to the boundary layer height, $h$. 
With increasing height, the spectral intensity of shear production subrange-generated turbulence diminishes with diminishing shear. In the next section, we engage in a more detailed analysis of the generation and scaling of the shear production subrange in the so-called "eddy-surface layer" embedded within the lowest one-third of the neutrally stratified surface layer.

In Section 3, we present spectra measured in the atmospheric surface layer from the met mast at Høvsøre; to demonstrate the extended subranges of $-1^{\text {st }}$-degree power spectral laws in support of Tchen's early (1953 - 1954) predictions, these are screened to contain only near-neutral condition spectra. In the atmospheric boundary layer, the largest eddies are also produced on scales proportional to the height of the boundary layer.

After being generated at scales $\lambda \approx h$, the largest boundary layer eddies interact nonlinearly and break up into smaller and smaller eddies, in a continuing cascading process, producing the well-known inertial subrange of $S(n)$ following a $-5 / 3^{\text {rd }}$-degree power law. This cascade process is responsible for the generation of the so-called inertial range, which is evident at all heights in the high frequency part of the spectra in Figure 2 and is of a different nature from the process following a similar power law in the mesoscale range. At even higher frequencies, the now very small eddies become comparable in size to the Kolmogorov microscale, which for atmospheric turbulence is on the order of millimetres, $O\left(10^{-3}\right)$, and enter the so-called dissipation region in which all turbulent fluctuations are eventually smeared out and the kinetic energy of the eddies are dissipated into heat by viscous dissipation (this is not apparent on the scales presented in Figure 2).

On a daily timescale, the boundary layer spectrum is strongly influenced by the thermal structure of the atmosphere, which can be alternately categorized as: 1 ) unstable, with the warmest conditions at the bottom (typical solar heating); 2) neutral, with no thermal influence; or 3) stable, in which conditions are coldest at the ground, typically as a result of night-time radiation cooling. In the boundary layer portion of the annually averaged spectra in Figure 2, the rather strong thermal effects during both unstable and stable conditions have been averaged out across the multiyear extent of the measurements. Unlike the boundary layer spectrum, the mesoscale spectrum appears to be generally relative insensitive to changes in the atmosphere's thermal structure $[7,8]$.

In unstable conditions, boundary layer turbulence is enhanced. This will typically happen at eddy scales of $\lambda \approx h$, or $n \approx u / h$, which is close to the lowest frequencies of the boundary layer portions of the spectra shown in Figure 2.

During strong convection, the low frequency portion of the spectrum might swamp the "spectral gap" [8]. The situation is reversed for stable conditions, in which the spectrum is diminished primarily at lower frequencies; in this case, the spectral peak also gradually erodes, causing the spectrum to appear to slide toward higher frequencies and revealing a continuation of the mesoscale spectrum at higher frequencies [8], [9] . The behavior of the spectra in the surface layer is well-described by the Monin-Obukhov hypothesis, in which the spectra are given as functions of $u, z$, and where atmospheric stability is parameterized by Monin-Obukhov similarity scaling in terms of dimensionless height $z / L$, where $L$ is the Monin-Obukhov length scale, and surface characteristics are parameterized via the surface roughness 
parameter $Z_{0}$, yielding velocity spectra for all three velocity components in the common form $(d u / d z)^{-2} S(n z / u, z / L)$. A notable exception in which such schematization is unavailable lies in those parts of the unstable-neutral spectra that depend on the convective mixing height, $z_{i}, \mathrm{cf}$. [9], [10], [11], [12].

\section{The Inertial and Shear production Subranges}

In this section, we provide a more thorough review and description of the physics behind the shear generation of turbulence in the shear production subrange and compare it with the well-known inertial subrange.

\subsection{Inertial Subrange}

It is well known that, under homogeneous and stationary turbulence, the spectra of the three wind velocity components satisfy the Kolmogorov-Obukhov $-5 / 3^{\text {rd }}$-degree power law in the inertial subrange of the wavenumber, $k$ (i.e., for $\eta^{-1} \gg k \gg z^{-1}$, where $\eta=\left(v^{3} / \varepsilon\right)^{1 / 4}$ is the Kolmogorov length scale, $\varepsilon$ is the mean energy dissipation rate, and $v$ the kinematic viscosity of air, cf., e.g., [13]).

The inertial subrange is characterized by an equilibrium between the shear-produced turbulent kinetic energy at lower wavenumbers, which is cascaded through the inertial subrange, and dissipation at higher wavenumbers [14],[15],[16]. In the inertial subrange, the turbulent kinetic energy spectrum obeys a $-5 / 3^{\text {rd }}$ power spectral law, $F_{11} \sim \varepsilon^{2 / 3} k_{1}^{-5 / 3}$, where $k_{1}$ is the wavenumber along the mean wind direction, which by application of Taylor's frozen turbulence hypothesis can be expressed by $k_{1}=2 \pi n / u$, where $n$ is the frequency in $\mathrm{Hz}$ and $u$ is the mean wind speed. $F_{11}\left(k_{1}\right)$ is the turbulent kinetic energy wavenumber spectrum of the velocity component $u$ along the mean wind direction, representing the kinetic energy associated with the wavenumbers between $k_{1}$ and $k_{1}+d k_{1}$.

Many analytical models have been proposed for the parameterization of spectra in near-neutral stratification. Two widely used atmospheric spectra parameterizations are the von Kármán [17] and Kaimal spectral models [18]. In 1990, Højstrup et al. extended the Kaimal spectrum by adding convective spectral energy to the unstable stratified boundary layer turbulence based on convective scaling (i.e., surface heat flux and convective boundary layer height) [12].

In addition, several 3D simulation codes for generation of isotropic turbulence have been developed. For simulation of neutrally stratified 3D wind fields in a weak-shear see e.g. the Mann turbulence model [19].

\subsection{Shear Production Subrange}

In the atmospheric surface layer, the vertical mean wind speed profile is well-known to be characterized by the logarithmic wind profile $u=u_{*} / \kappa \ln \left(z / z_{0}\right)$. The corresponding vertical wind shear, $u^{\prime}=u_{*} /(\kappa z)$, is 
inversely proportional to height and can become nominally large near the ground. From a scaling point of view, the shear stress velocity $u_{*}$ has been proposed as the dominant scaling parameter in strong sheardriven turbulence in place of the dissipation rate $\varepsilon$ (cf. [20]).

In the atmospheric boundary layer, the dissipation rate used for scaling the inertial subrange spectra is of the order $\approx u_{*}^{3} / z$. Near the ground, however, the dissipation rate becomes a strong non-constant function of height, which disqualifies it here as a proper scaling parameter for turbulence near the ground.

In the lowest one-third of the atmospheric surface layer-the so-called "eddy surface layer" (cf. [2]), also called the "eddy shear layer" [21] - the generation of turbulent kinetic energy by velocity wind shear is strong and, to first-order, inversely proportional to the height above ground.

An early theoretical investigation of the energy transfer across the spectrum was developed by Tchen[5,6], who investigated the transport process of turbulent energy in terms of the energy transfer functions originally proposed by Obukhov in 1941 [16] and Heisenberg in 1948 [16].

In a moderate or weak shear flow, as encountered in the atmospheric boundary layer in say the upper twothirds of the surface layer, the spectral energy transfer function $W_{k}=2 v_{k} \int_{0}^{k} d k^{\prime} k^{2} F\left(k^{\prime}\right)$ dominates the transfer of energy from wavenumbers smaller than $k$ to wavenumbers larger than $k$. The quantity $v_{k}$ denotes the turbulent viscosity. In this part of the atmosphere the cascade of energy is predominantly balanced between spectral energy transfer and dissipation; hence the energy spectrum for a moderate shear flow predicted by Tchen is the same as that given by Heisenberg's and Obukhov's theories, i.e., the usual $-5 / 3^{\text {rd }}$-power law, $F_{11} \simeq \varepsilon^{2 / 3} k_{1}^{-5 / 3}$.

However, in a strong shear flow the spectral transfer across wavenumbers can become negligible compared to the energy locally generated by strong shear, $W_{k} \ll u^{2}=\left(d u_{i} d x_{j}\right)^{2}$ and, according to Tchen's prediction ([6] Eq. 33), the dissipation will in this case be balanced by shear production , viz.:

$\varepsilon \approx v_{k}\left[u^{\prime 2} 2 \int_{0}^{k} d k^{\prime} k^{\prime 2} F\left(k^{\prime}\right)\right]^{1 / 2}$.

It follows that the shear production subrange spectrum in a strong shear flow, i.e., for large $u^{\prime}$, is of the form $F=\left(\varepsilon / \kappa u^{\prime}\right) k^{-1}$, cf. Tchen 1953 [5], Eqs 17 and Tchen 1954 [6], Eq. 34.

For the neutral atmospheric surface layer, estimating the dissipation rate as $\varepsilon \simeq u_{*}^{3} /(\kappa z)$ and having a mean vertical shear $u^{\prime}=u_{*} /(\kappa z)$, Tchen's turbulence theory predicted the existence of a shear production dominated energy spectrum, for the wind component parallel with the mean wind direction, in a strong shear-dominated subrange, to be of the form

$$
F \sim u_{*}^{2} k^{-1}
$$

In summary, dimensional analysis [20] and Tchen's early theoretical work based on spectral transfer function theory predict that a shear spectrum with a $-1^{\text {st }}$-degree power law characterizes the wind spectrum of turbulence of the longitudinal velocity component in a strong velocity shear. According to Tchen, a "shear production subrange" is applicable when the turbulent coherent structures or eddies are 
larger than the height of observation above the surface, i.e., for a subrange of wavenumbers such that $k z<1$, where $z$ is the observation height.

\subsection{Atmospheric boundary layer spectra within the eddy surface layer}

Several studies [20] refer to laboratory measurements carried out by Perry and Abell in pipe-flow turbulence in which a region near the wall showed clear evidence of a $-1^{\text {st }}$-degree power law [22-24] in a spectral subrange in where $k z<1$. In addition, [25] developed a wall-wake model for strong shear turbulence based on Townsend's 1976 "attached eddy hypothesis" [26].

Spectra of the three wind components measured by a sonic anemometer at $10 \mathrm{~m}$ height above the ground during the 1984 surface layer Borex puff diffusion trials provided also a clear experimental evidence of a $1^{\text {st }}$-degree power law shear production subrange adjacent to a $-5 / 3^{\text {rd }}$-degree inertial subrange in stably stratified atmospheric shear flow [4]. An inverse linear power law was observed to extend almost a full decade in both of the two horizontal wind component spectra in the wavenumber range between $1 / z$ and $0.1 / z$.

Högström, Hunt and Smedman [3] investigated theories and measurements for turbulence spectra and variances in the atmospheric neutral surface layer. Their approach followed the inactive turbulence concept first introduced by Townsend in 1961, according to which the turbulence motion near the ground comprises: 1 ) an active part that produces stress, and 2) an inactive part that arises in the upper part of the boundary layer that does not contribute to the shear stress. Atmospheric surface layer spectral measurements show a mean longitudinal velocity spectrum from the "Laban's Mills' site [3]. This spectrum shows clear evidence of four out of the five spectral ranges (i) through (iv) listed below.

For the lowest one-third of the surface layer-the eddy surface layer-it is stated that the low wavenumber spectrum for the longitudinal wind component is strongly distorted by blocking, intense shearing, and surface scrapping mechanisms.

With reference to [23], the longitudinal velocity spectrum $F_{11}\left(k_{1}\right)$ exhibits five characteristic spectral regimes for the boundary layer turbulence, cf. [3] and [7,8]:

(i) For $k_{1} \gg z^{-1}$, it follows the Kolmogoroff inertial range, with spectral slope $-5 / 3$

(ii) For $\Lambda_{s}^{-1}<k_{1}<<z^{-1}$ it follows the eddy surface range; for $\Lambda_{s}^{-1} \ll k_{1}$, where $\Lambda_{s}$ is the largest horizontal eddy there is a self-similar range (cf. the shear production range) where $F_{11}\left(k_{1}\right) \propto \gamma u_{*}^{2} k_{1}^{-1}$. The constant of proportionality $\gamma$ is of order unity. In frequency representation, this subrange can be given by $\frac{n S_{u}(n)}{u_{*}^{2}} \approx \gamma$, where $\gamma \sim 1$ and $S_{u}(n)$ is the longitudinal frequency spectrum and $n$ is the frequency in $\mathrm{Hz}$.

(iii) Below the shear production subrange, i.e., for wavenumbers $k_{1}<\Delta_{s}^{-1} \sim \frac{u_{*}}{u} h$, where $h$ is the height of the atmospheric boundary layer, the spectrum $F_{11}\left(k_{1}\right)$ "flattens", i.e., it has a "zero" power law, as it approaches its lowest limit for a random stationary process with a single outer length scale $\Lambda_{s}$ in accordance with the Wiener-Khintchine theorem, $F_{11}\left(k_{1} \rightarrow 0\right) \propto u_{*}^{2} \Lambda_{s}$. 
As investigated by [23], [25], and [2], by applying Taylor's frozen turbulence hypothesis $n / k_{1}=u$, this "flat" subrange becomes, in frequency form, $\quad k_{1} F_{11}\left(k_{1}\right) / u_{*}^{2}=n S_{u}(n) / u_{*}^{2}=\Lambda_{s} n / u$

Now, with reference to [6], $\Lambda_{s}$ in an atmospheric boundary layer flow is of the order of (three times) the boundary layer depth $h \sim 0.2 u_{*} / f_{C}$, where $f_{C}[\mathrm{~Hz}]$ is the Coriolis parameter. Thus, we substitute

$$
n S_{u}(n) / u_{*}^{2}=\frac{A u_{*}}{f_{c}} \frac{n}{u} ; A \sim 0.6
$$

Again, according to [6], the parameter $A$ may vary as a function of height $A=g(z)$, where $g(z)$ is a function to be determined from measurement.

The first three subranges (i)-(iii) are shown schematically in Figure 3.

(iv) In a subrange at even lower frequencies from approximately $\sim 0.2 \mathrm{day}^{-1}$ to $\sim 1 \mathrm{hr}^{-1}$ and separated from subranges (i)-(iii) by a "spectral gap" [8], the eddies are characterized by large two-dimensional horizontal mesoscale structures. Aircraft measurements by [27] showed also that tropospheric turbulence contains a $-5 / 3$ subrange in the planetary boundary layers for two-dimensional structures on scales of up to about $400 \mathrm{~km}$ in horizontal extent.

(v) As shown in [7], on even larger scales of $400-4,000 \mathrm{~km}$ in horizontal extent aircraft data observation [26] revealed the existence of an enstrophy (a type of potential density related to vorticity) cascade subrange with a spectral power law of -3 . Similarly, on these tropospheric scales spectral subranges following both $-5 / 3^{\text {rd }}$ and $-3^{\text {rd }}$ power laws could also be found via GCM re-analysis.

Notably, the early (1954) theory of Tchen also predicted a "buoyancy subrange" with a $-3^{\text {rd }}$ power law for stable stratified turbulence. This has been compared to a large-scale anisotropic spectrum of form $N^{2} k^{-3}$, where $N$ is the Brunt-Väisälä frequency, cf. [4]. 


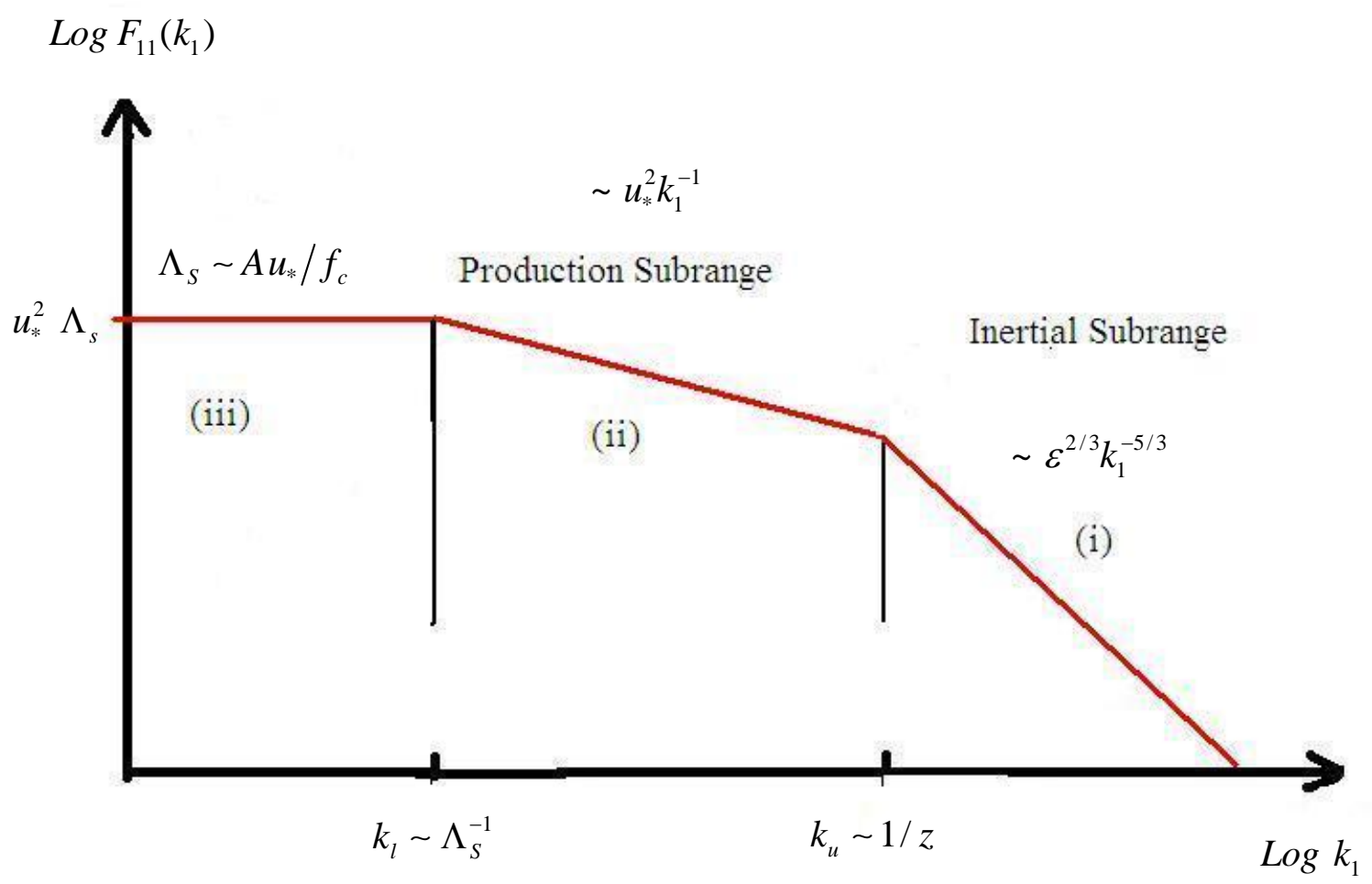

Fig. 3. The three spectral regimes of boundary layer wind speed turbulence characterized by [3]: (i) Inertial subrange with spectral law -5/3; (ii) shear production subrange in the eddy surface layer characterized by strong shear close to the surface (also denoted as "the eddy shear layer") with an inverse linear (degree -1) spectral power law; and (iii) a "flat" subrange of "zero" power spectral law characteristic of a component spectrum of turbulence with a single outer length scale $\Lambda_{s}$ 


\section{Parameterization of the shear production subrange}

\subsection{Determining the shear production subrange's upper and lower bounds $f_{u}$ and}

$f_{l}$

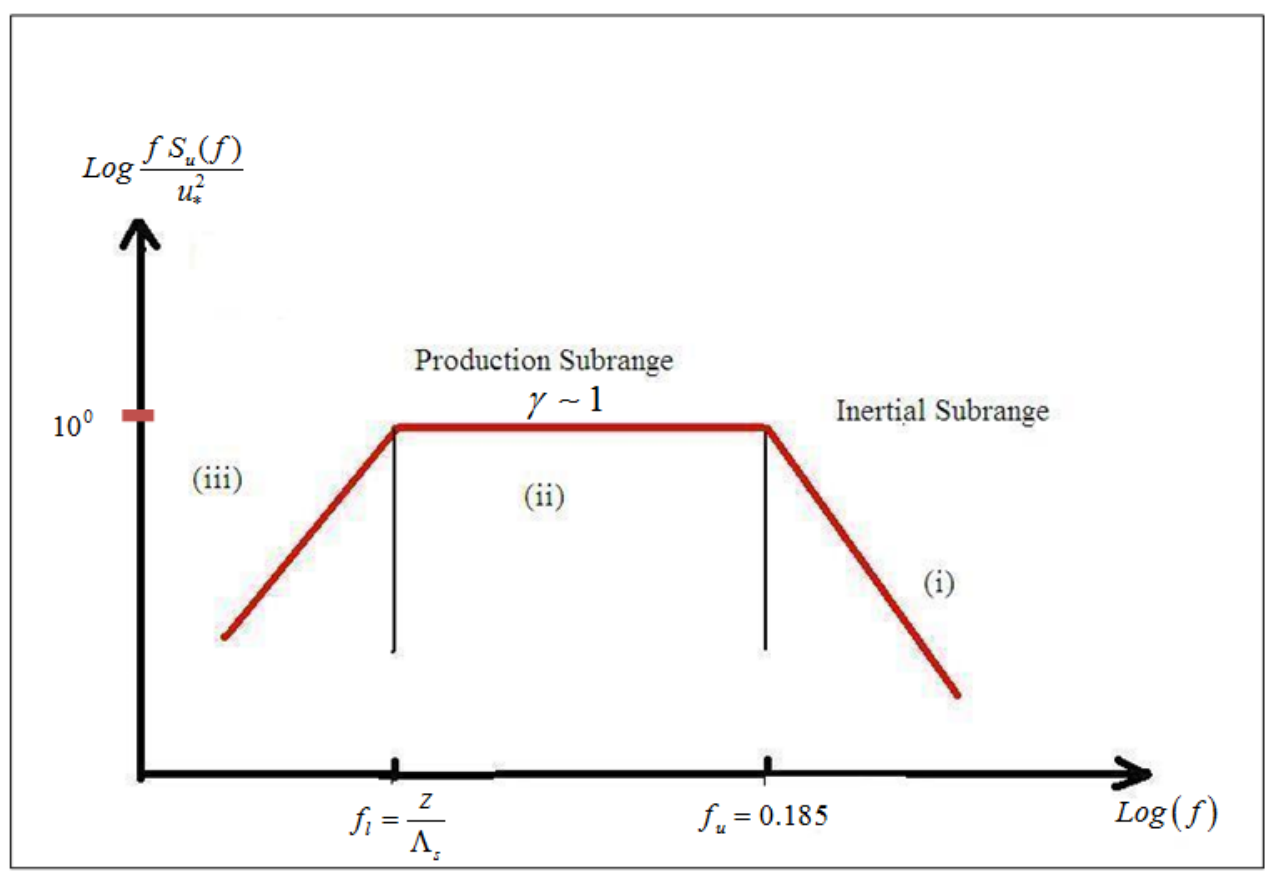

Figure 4. Enhanced spectral model combining (i)-(iii) as functions of dimensionless frequency $f=n z / u$ : (i) inertial subrange, (ii) shear production subrange, and (iii) "flat" spectral range. Subrange (ii) extends the classical Kaimal spectrum model with a shear production subrange between the dimensionless peak frequency $f_{u}$ and the shear production subrange low frequency limit $f_{l}$.

Figure 3 shows schematically the shear-production enhanced spectrum embedded between the inertial subrange (i) and the flat part of the Kaimal model spectrum (iii) in terms of non-dimensional frequencies, $f$ . In [3], it was demonstrated that the constant spectral intensity $\gamma$ of the shear production subrange is of the order of unity, as discussed in section 2.3. Hence, given the spectral amplitude in the shear production subrange, $\gamma \sim 1$ and applying the Kolmogoroff spectrum to the inertial subrange with a longitudinal Kolmogoroff constant $\alpha_{1}=0.52$, the intersection between (i) and (ii) sets the upper bound of the shear production subrange as $f_{u}=n_{u} \frac{Z}{u}=0.185$, cf. [3].

Again, by assuming $\gamma \sim 1$, the intersection between subranges (ii) and (iii) determines the lower-bound frequency. From subrange (iii), with $n S_{u}(n) / u_{*}^{2}=\Lambda_{s} n / u$ set to unity for $n=n_{l}$ and setting $\Lambda_{s}=A u_{*} / f_{c}$ with $A=0.6$ according to [3], the non-dimensional lower frequency bound of the shear production subrange becomes $f_{l}=n_{l} \frac{Z}{u}=\frac{f_{c} z}{0.6 u_{*}}$. 


\subsection{A combined inertial subrange and shear production subrange spectral model}

A simple analytic spectral model that extends the classical Kaimal spectrum for neutrally stratified surface layer turbulence by including a parameterization of the production subrange and is applicable to the surface layer and the eddy surface layer near the ground was proposed in [28]. Subsequently, the spectral model was compared to raw unscaled velocity spectra measured in the meteorological mast at Høvsøre [29].

This model extends the inertial subrange spectrum model of Kaimal by adding a shear production subrange with a $-1^{\text {st }}$-degree power law to the lower part of the surface layer within the eddy surface layer. In this manner, Kaimal et al.'s well-known longitudinal model spectrum could be extended with a shear production subrange below its classical peak frequency.

The starting point is the neutral spectral model by Kaimal et al. [18]. According to [10], the neutral longitudinal spectrum is of the form

$$
\frac{n S_{u}(n)}{u_{*}^{2}}=\frac{d f}{(1+e f)^{5 / 3}} \text {, where } d=105, e=33 \text { and } f=n z / u \text {. }
$$

Next, we extend the neutral Kaimal spectrum model with a $-1^{\text {st }}$-degree power law to include the "shear production subrange" of the eddy surface layer within the bounds $f_{u}$ and $f_{l}$. The enhanced spectrum model in non-dimensional analytical form as a function of non-dimensional frequency $f$, is

$$
\frac{n S_{u}(n)}{u_{*}^{2}}=\frac{a f / f_{l}}{\left(1+f / f_{l}\right)\left(1+f / f_{u}\right)^{2 / 3}}
$$

where the parameter $a$ is a scale parameter for the spectral intensity, which can be determined by matching the spectral intensities of the two model spectra in the high-frequency limit. From Eq. (3.1), $\operatorname{Lim}_{f \rightarrow \infty} n S_{u}(n) / u_{*}^{2}=d e^{-5 / 3} f^{-2 / 3}$. Similarly, from Eq. (3.2), $\operatorname{Lim}_{f \rightarrow \infty} n S_{u}(n) / u_{*}^{2}=a f_{u}^{2 / 3} f^{-2 / 3}$, and by matching we can determine $a=d e^{-5 / 3} f_{u}^{-2 / 3}=0.31 f_{u}^{-2 / 3}$. In summary, the shear production subrange extended spectrum model, with its scaling parameters, becomes

$$
\begin{aligned}
& \frac{n S_{u}(n)}{u_{*}^{2}}=\frac{a f / f_{l}}{\left(1+f / f_{l}\right)\left(1+f / f_{u}\right)^{2 / 3}} ; \\
& \text { with } \\
& a=0.953 ; f_{u}=0.185 \text { and } f_{l}=f_{c} z / 0.6 u_{*}
\end{aligned}
$$

Note that $f$ is the non-dimensional frequency, $f=n z / u$, and the lower frequency limit, $f_{l} \simeq z / 3 h$, scales with measurement height $z$ over three times the neutral boundary layer height, $h$. The extended spectrum model for the wind component in the mean wind direction applies to near-neutral surface layer longitudinal spectra and includes the shear production subrange of the eddy surface layer in the $1 / 3$ lowest part of the atmospheric surface layer.

Unlike Kaimal's spectrum model, the extended spectrum model does not collapse into a single universal function independent of height; the measurement height $z$ itself becomes an additional important scaling parameter. The extended spectrum model (3.3) is shown in Figure 5. As is seen, it represents a smooth analytical form of the three subranges (i), (ii), and (iii) from Figures 3 and 4. 


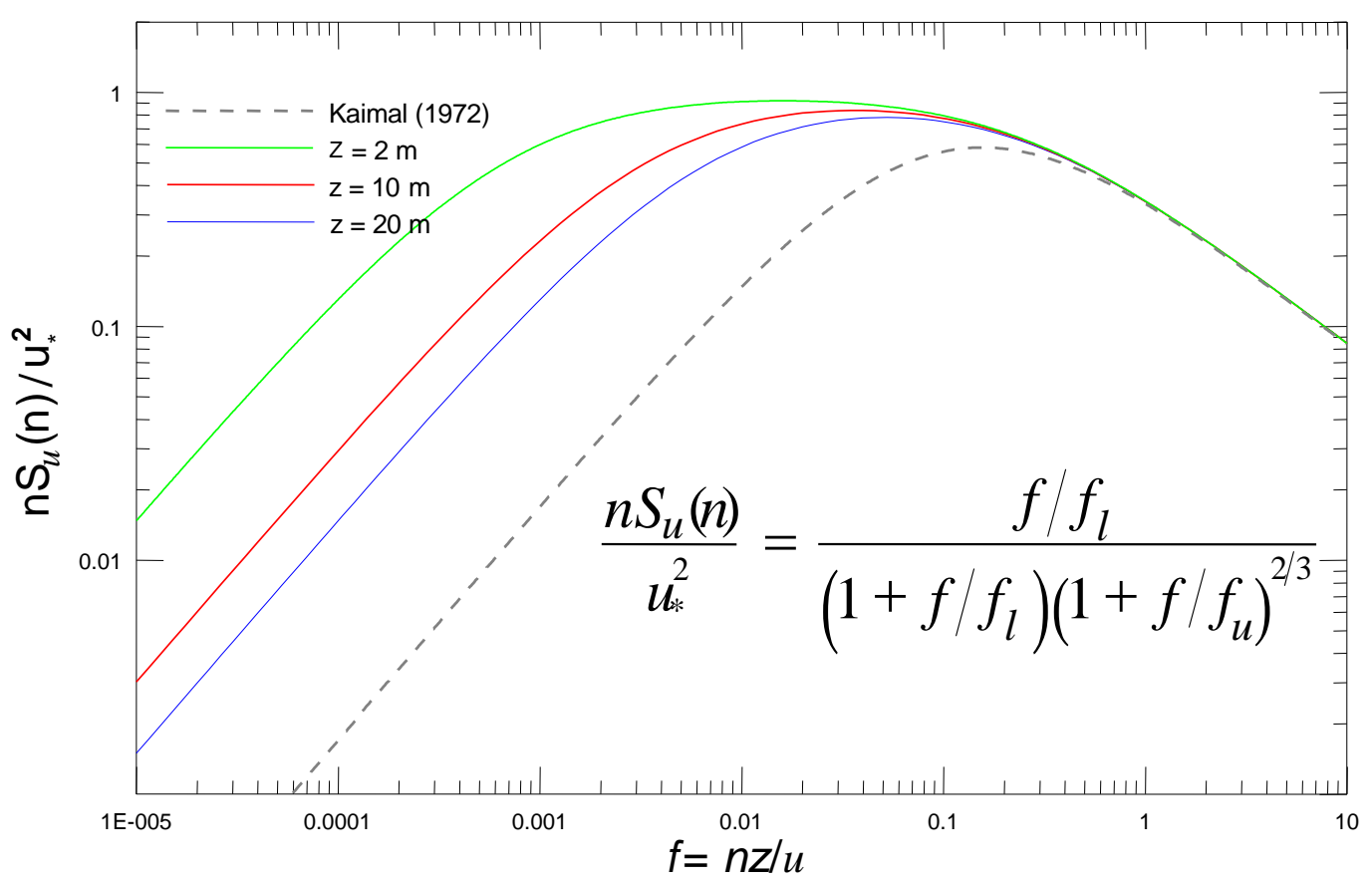

Figure 5. Mikkelsen et al. (2001) extended spectrum model [Eq. (3.3)] at heights: $2 \mathrm{~m}$ (green line), 10m (red line), and $20 \mathrm{~m}$ (blue line). For comparison, the unmodified Kaimal model spectrum [Eq. (3.1)] is also shown (black dotted line).

\section{Comparison with Spectral Measurements}

\subsection{Atmospheric Surface layer Spectral Measurements}

\section{Case 1: Stable atmospheric surface layer spectra}

An early attempt to compare measurements of wind spectra with his various subrange theories was published by Tchen et al. in Physica Scripta in 1985 [4] (cf. Figure 1). In the early 1980s, wind component spectra were calculated from wind records measured in the eddy surface layer at $10 \mathrm{~m}$ height as part of the Borex smoke diffusion field trials conducted over flat terrain at the Borris Moors in Western Jutland, Denmark [30] [31].

Figure 1 on page 616 in [4] shows all three wind component spectra calculated from a one-hour measurement period sampled at $20 \mathrm{~Hz}$ by a three-axis ultrasonic anemometer (Kayo Denki type DAT 310) installed on top of a $10 \mathrm{~m}$ meteorological mast. These spectra were recorded following a transition to stable stratification in the hour before midnight on August 21, 1984. 
As is characteristic of low wind speed conditions during moderately stable atmospheric conditions ( $z / L=0.2$ ), the mean wind speed was only $u=2.54 \mathrm{~ms}^{-1}$ and the friction velocity was $u_{*} \simeq 0.13 \mathrm{~ms}^{-1}$. The spectral portions in the shear production subrange therefore reflected no contributions from convective generated turbulence. It should be noted that the spectra in this figure are multiplied by frequency, flattening the shear production subrange (i.e., the shear production subrange is presented as a horizontal line (S) with a "zero-power" spectral law). The three subranges proposed by Tchen in 1954-I, S, and $\mathrm{G}-$ are identified, and the measurements show evidence of the three predicted subranges$k^{-5 / 3}, \mathrm{k}^{-1}, \mathrm{k}^{-3}$-for inertia, shear production, and buoyancy, respectively [4].

The Borex data set from 1984 provides, to our knowledge, the first full-scale atmospheric surface layer experiment producing evidence of a $-1^{\text {st }}$-degree power law, indicative of a separate shear production subrange, adjacent to the $-5 / 3^{\text {rd }}$ inertial subrange. A sub-range with a power law of -1 was found to extend almost one full decade in both of the two horizontal wind component spectra in the wavenumber range between $1.0 / \mathrm{z}$ and $0.1 / \mathrm{z} \mathrm{m}^{-1}$.

\section{Case 2: Unstable atmospheric surface layer spectra}

A subsequent work ([32], page 322) compared an ensemble of four transverse (v-component) spectra measured as described above at $10 \mathrm{~m}$ height during the 1981 Borex diffusion trials. The spectra were calculated from time series measured during Borex 81 Run 1B by four identical Kayo Denki sonic anemometers installed on top of a row of four met towers separated by between 4.5 and $30 \mathrm{~m}$ horizontally [30]. In this case, however, the atmosphere was unstable $(z / L=-0.11)$ with a mean wind speed $u=5.93 \mathrm{~ms}^{-1}$, a friction velocity $u^{*}=0.44 \mathrm{~ms}^{-1}$, a Monin-Obukhov length $L=-90 \mathrm{~m}$, and a convective boundary layer height $h$ of $950 \mathrm{~m}$, cf. [32].Similar to the spectra in Case 1, the spectra presented in [32] also show evidence of: (i) an inertial subrange in the interval: $0.25<k<3.8\left[m^{-1}\right]$; (ii) a shear production subrange in the interval $0.01<k<0.25\left[\mathrm{~m}^{-1}\right]$; and (iii) a buoyance subrange in the interval: $0.004<k<0.01\left[\mathrm{~m}^{-1}\right]$.

However, as these spectra were observed during slightly unstable atmospheric conditions, the spectra with a more or less flat subrange in the interval between $0.01<k<0.25\left[\mathrm{~m}^{-1}\right]$ might have been masked and contributed to by mixed-layer spectral energy caused by heat convection in the same subrange range during unstable atmospheric stratification, cf. [10].

\section{Case 3: Neutral atmospheric surface layer spectra during high wind speed}

To reduce the influence of atmospheric stratification and stability on the eddy surface layer turbulence, only spectra measured during near-neutral atmospheric conditions are considered in the following.

The spectra of turbulence in a near-neutral stratified atmosphere, which occurs in the part of the surface layer in which the wind shear is moderate, have successfully been parameterized by surface layer scaling based on surface friction velocity and using the measurement height $z$ as the dominant length scale involved (cf. Eq. (3.1) and [18]). 
For instance, [12] presented neutral spectral measurements of the longitudinal wind component from a 64 $\mathrm{m}$ tall meteorological mast equipped with cup anemometers at $3,10,25,45,56$, and $64 \mathrm{~m}$ located at Nibe Bredding in northern Jutland, Denmark, which was used extensively for measurements in the 1980s. The meteorological mast was installed at a coastal site on a shallow beach that was flooded during the strong westerly winds measured during the experiment. The upwind fetch over water for westerly flow was approximately $16 \mathrm{~km}$.

Figure 2 in [12] provides neutral longitudinal frequency spectra measured at heights 10, 25, 45, and $56 \mathrm{~m}$ of $n S_{u}(n)$ as functions of frequency $n$ for strong wind speeds $u_{10 m}=23 \mathrm{~m} / \mathrm{s}$ and during a near-neutral stratification $\left(u_{*}=1.1 \mathrm{~ms}^{-1} ; \quad|L|>600 \mathrm{~m}\right.$ ). By fitting straight lines with power laws of $-5 / 3,-1$, and "zero," respectively, distinct subranges of: (i) Inertial subrange, (ii) shear production, and (iii) the flat part can be identified.

When the Nibe spectra were plotted against the non-dimensional frequency, the Nibe spectra successfully collapsed in the inertial subrange according to Kaimal et al.'s similarity theory [17]. Højstrup et al. 1990 also observed that the low-frequency parts of the spectra, i.e., the parts of the spectra below the peak frequency in Kaimal's model spectrum, appeared to be fairly independent of the measurement height. This is in accordance with the shear production subrange scaling properties, which, in contrast to the Kaimal scaled spectrum, which also involves measurement height, depends only on friction velocity $u_{*}$, a quantity that is considered approximately constant with measurement height in the eddy surface layer close to the ground.

From the flat subrange (iii) of the Nibe Run 1 spectrum in Figure 2 of [12], we have, in accordance with [6], inferred the constant $A \simeq 0.6$.

Case 4: Høvsøre spectra in the height range 10-100 m during near-neutral atmospheric stability conditions

Ensemble-averaged near-neutral atmospheric surface layer spectra were calculated from six sonic anemometers installed in the meteorological reference mast established at the Danish test station for large wind turbines in $\mathrm{H} \varnothing \mathrm{v}$ s $\varnothing \mathrm{re}$, Western Jutland, cf. Figure 1. The spectral measurements presented below were sampled at $20 \mathrm{~Hz}$ by six sonic anemometers installed in the met mast at heights of 10, 20,40,60, 80, and $100 \mathrm{~m}$ from April 7 through December of 2005.

The spectral measurements are binned into an East sector, representing a homogeneous upwind fetch within the wind direction sector 75 to 105 degrees, and a West sector with wind directions in the range 255-285 degrees and representing an inhomogeneous coastal inflow sector. In the latter case, an internal boundary layer develops from the nearby coastline approximately $1.5-2 \mathrm{~km}$ upwind. The development of an internal boundary layer from the coastline is consequently expected to influence the measurements from approximately $60 \mathrm{~m}$ upwards in this sector. 
Nine month of ensemble averaged spectra of the longitudinal wind turbulence measured by six Metex USA1-type F2901A sonic anemometers installed at heights of 10, 20, 40,60, 80, and $100 \mathrm{~m}$ in the $116.5 \mathrm{~m}$ tall meteorological mast at $\mathrm{H} \varnothing \mathrm{v} \varnothing$ re, Denmark [1] are presented in Figures 6a and 6b.

Time series of the three wind components were sampled at $20 \mathrm{~Hz}$, followed by linear trend removal. The spectra were then processed and binned according to height and wind direction sector. In addition, we considered the two sides of near-neutral atmospheric stability: 1 ) the near-neutral unstable atmospheric stability cases $-0.1<z / L<0$ representing "near-neutral but on the unstable side," and 2 ) the nearneutral but stable side of stability $0<z / L<0.1$ representative of "near-neutral spectra approaching neutral from the stable side." The stability parameter $z / L$ is determined from the sonic measured heat flux and friction velocity at each measurement height. The number of ensemble averaged spectra per height is indicated by $\# x x \#$, where $x x$ is the number of spectra in the ensemble.

The ensemble-averaged spectra have been binned as functions of measurement height and by wind direction sector and near-neutral stability sector. Scaled by the ensemble averaged friction velocity squared, $u_{*}^{2}$, and the dimensionless dissipation rate, $\phi_{\varepsilon}=\varepsilon \kappa z / u_{*}^{3}$, the Høvsøre measured longitudinal velocity component spectra $S_{u u}$ are presented as normalized frequency spectra, $n S_{u u}(n) /\left(u_{*}^{2} \phi_{\varepsilon}^{2 / 3}\right)$, plotted as functions of non-dimensional frequency $f=n z / u[-]$. The left column shows spectra from the six measurement heights in the homogeneous East sector, while the right column shows corresponding spectra with wind coming from the coast in the West sector. 
Høvsøre Spectra at heights 10 - $100 \mathrm{~m}$

Unstable side of near neutral: $-0.1<z / L<0$
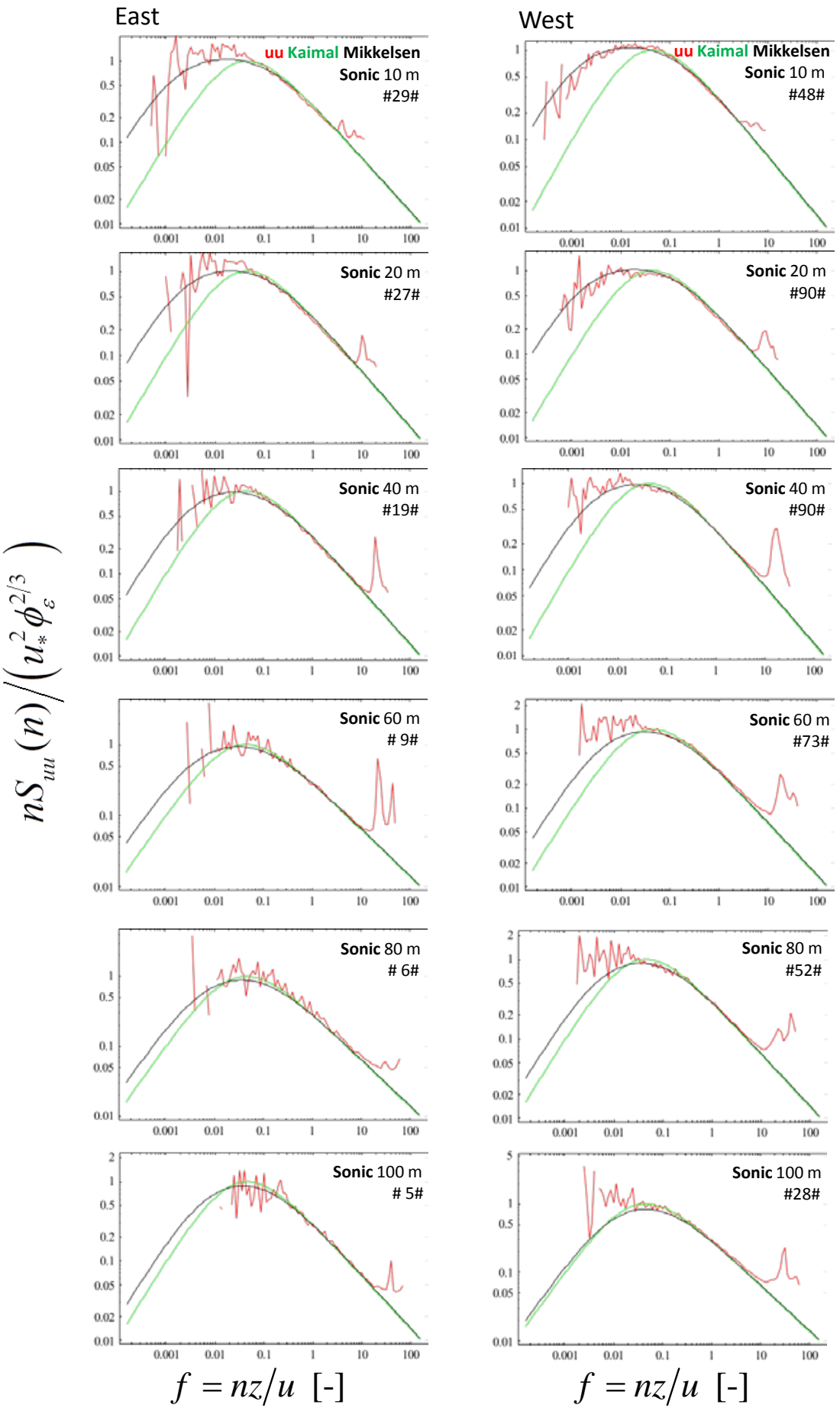

Figure 6a. Ensemble-averaged longitudinal wind component energy spectra uu (red lines) measured by sonic anemometers in 1) the eastern wind direction sector (left column), and 2) the western wind direction sector (right column), at six heights during near-neutral unstable stability. The numbers between \#\# are the ensemble size per spectrum. Kaimal's classical spectrum model [Eq.(3.1)]: green line. Mikkelsen et al. (2001)'s production subrange extended spectrum model [Eq. (3.3)]: black line. 
Høvsøre Spectra 10 - 100 m

Stable side of near neutral: $0<z / L<0.1$
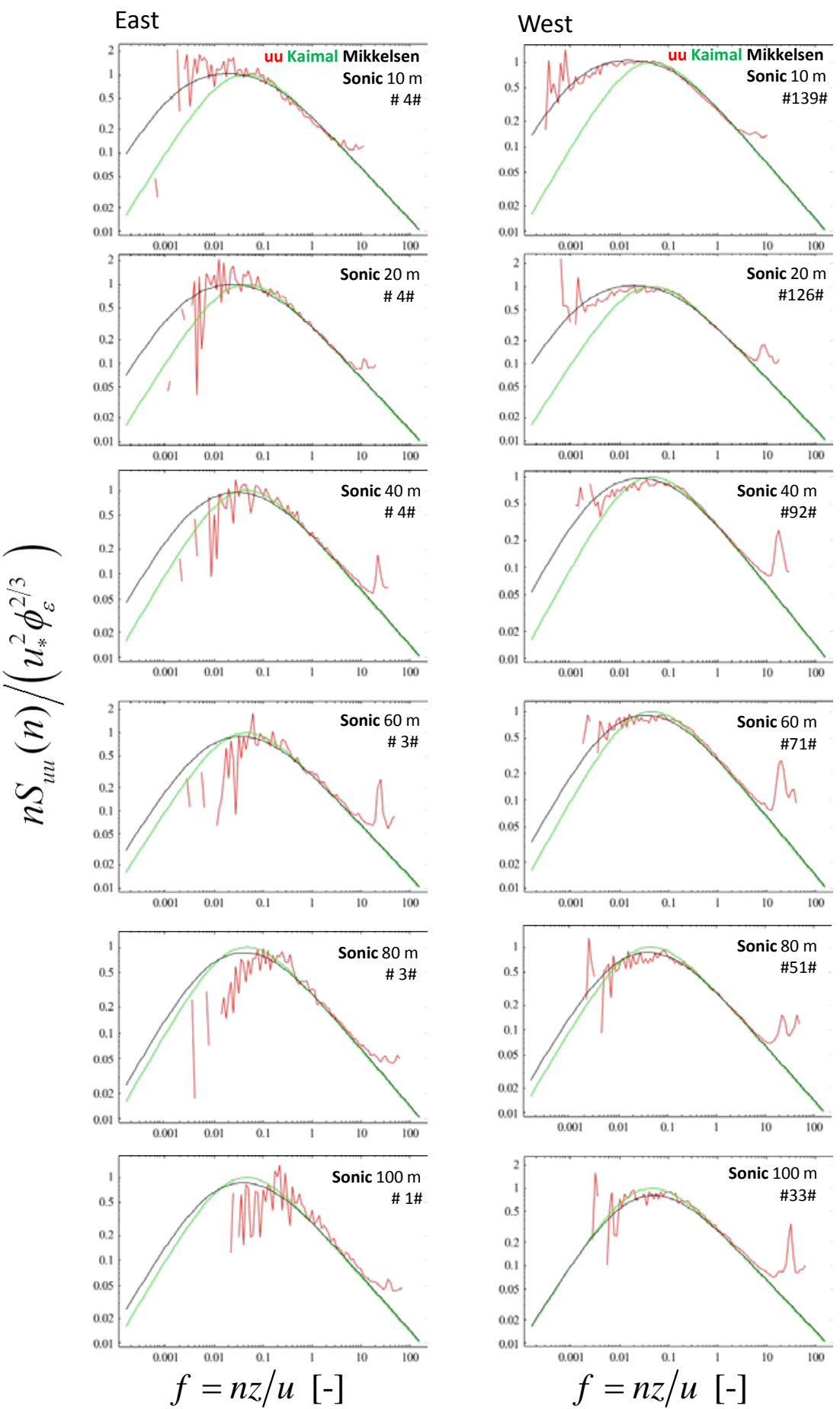

Figure 6b. Ensemble-averaged longitudinal wind component energy spectra uu (red line) measured by sonic anemometers in 1) the eastern wind direction sector (left column), and 2) the western wind direction sector (right column), at six heights during near-neutral stable stability. The numbers between \#\# are the ensemble size per spectrum. Kaimal's classical spectrum model (3.1): green line. Mikkelsen et al. (2001)'s production subrange extended spectrum model (3.3): black line. 
The $H \varnothing v$ s $\varnothing$ re spectra show clear evidence of distinct $-5 / 3^{\text {rd }}$-degree power law inertial subranges at all measurement heights and irrespective of wind direction and atmospheric stability.

Unlike the $H \varnothing v s \varnothing r e$ spectra previously presented in [29], the sonic measured ensemble-averaged friction velocities, $u_{*}$, have been re-normalized by height and sector, in some cases by $5-10 \%$, to merge the sonic anemometer-measured inertial subrange intensities with the inertial subrange of Kaimal's spectral model for neutral stratification [Eq. (1.3)]. While this intensity adjustment has re-scaled some of the spectral intensities presented in Figure 6, in particular for spectra measured in the West sector at heights of $60 \mathrm{~m}$ and above, it has not altered the measured spectral forms.

The spectra nearest the ground, i.e., the spectra at the lower heights of 10,20 , and $40 \mathrm{~m}$, show a significantly higher spectral intensity at frequencies below $0.1 \mathrm{~Hz}$, corresponding to the Kaimal spectrum model's peak frequency.

An enhanced turbulence level shows as a nearly flat spectral subrange in the frequency-multiplied spectrum $n S(n)$; this enhanced turbulence level represents, we believe, the shear production subrange of the near-neutral stratified eddy surface layer. From the spectra shown in Figures $6 a$ and $6 \mathrm{~b}$, the eddy surface layer appears extend to heights up to about $40 \mathrm{~m}$ above the ground.

It is also notable that the number of ensembles encountered within the considered fixed stability range $0<|z / L|<0.1$ decreases with measurement height, $z$, especially for the stable side of near-neutral spectra considered. The near-neutral stable spectrum at $100 \mathrm{~m}$ height in the East sector only contains one spectrum.

At the high-end of the non-dimensional frequency range, from $f \approx 10$ and above, several spectra show spurious peaks, which are an artifact of the sonic anemometer measurements at these high frequencies.

The Mikkelsen et al. (2001) production subrange extended spectrum model [Eq. (3.3)] is also added to the Høvsøre spectra shown in Figures $6 \mathrm{a}$ and $6 \mathrm{~b}$ together with the corresponding classical Kaimal spectrum model (3.1). The near-neutral dimensionless dissipation rate of 1.24 is accounted for via the spectral models' common inertial subrange intensity scale parameter, $d e^{-5 / 3} \simeq 0.31$.

For measurement heights above $40 \mathrm{~m}$, in particular for the spectra in the West sector, increased turbulence intensity can also be observed at low frequencies; however the increase relative to both spectral model predictions at this height is likely caused by the inhomogeneous intersection between the coastal marine boundary layer aloft and the internal boundary layer grooving up from the surface at the coastline and intersecting the $H \varnothing v s \varnothing r e$ mast at these heights in the West sector.

In Figure 6a and $6 \mathrm{~b}$ the extended spectrum model [Eq. (3.3)] is plotted as function of non-dimensional frequency $f=n z / u[-]$ in the interval $10^{-4}$ to $10^{2}$. However, due to the $20 \mathrm{~Hz}$ fixed sampling frequency and the 1-Hour sampling period applied, the spectral measurements presented are limited in frequency range from $1 / 3600$ to $10[\mathrm{~Hz}]$. When plotted in Figure 6 as function of non-dimensional frequency the spectral measurements cover only the limited non-dimensional frequency interval shown. Consequently, 
the study's experimental evaluation based on spectra measured at Høvsøre is limited to this frequency range.

\section{Discussion}

We have investigated previous and new measurements of the longitudinal wind component spectra observed during near-neutral atmospheric stability conditions. Based on these observations, we extended the classical Kaimal spectrum model for the neutrally stratified surface layer with a shear production subrange applicable for the near-ground eddy surface layer subrange.

The spectral measurements at Nibe and at $\mathrm{H} \varnothing \mathrm{v} \varnothing$ re show that a shear production subrange can become dominant in turbulence near the ground and can extend the surface layer spectrum with a shear production subrange in the wavenumber range in which eddy sizes are comparable to and larger than the measurement height above the ground.

With increasing height (starting at heights of 40-60 $\mathrm{m}$ above the ground) and diminishing vertical mean wind shear, the influence of the eddy surface layer shear production subrange vanishes.

However, closer to the ground (particularly at heights of 10-20 m and below) the influence of the shear production subrange on the neutral longitudinal velocity spectrum is significant, with the consequence that the otherwise dominant $-5 / 3$ inertial subrange for wavenumbers in the interval $\Lambda_{s}^{-1}<k_{1}<<z^{-1}$ become dominated by C.M Tchen's suggested $-1^{\text {st }}$-degree power law production subrange.

In our quest to obtain a better comprehension of atmospheric boundary layer turbulence, our previous understanding of the physics behind the classical Kaimal spectrum may have been somewhat disrupted for near-ground, near-neutral surface layer turbulence.

Investigations of the effects of wind loads from turbulence on, for instance, wind turbines usually assume that the inflow is well-described by inertial subrange turbulence characterized by a $k^{-5 / 3}$ spectrum; however, we have shown that part of the spectrum nearest to the ground is not scalable solely by the classical Kaimal inertial subrange spectrum model given in Eq. (3.1) .

In the current generation of huge multi MW turbines-particularly those used in offshore installations-the rotor diameters are bigger than the turbine hub height. Consequently, during revolution the blade tips soar through the eddy surface layer, the surface layer, and, sometimes, into the boundary layer above the surface layer.

Suspension bridges, air pollution dispersion, and huge wind turbines all operate or occur in the lowest part of the surface layer, in which a significant part of the turbulence is produced by strong wind shear production subrange turbulence. Notably, Tchen predicted the existence of the shear production subrange with characteristic spectral form $\sim u_{*}^{2} k^{-1}$ already in $1953-1954[5,6]$.

The suggested extended spectral model, Eq. (3.3), offers a simple analytical parameterization of the combined shear production and the classical inertia subrange turbulence and can be used in wind 
engineering applications and atmospheric dispersion modeling of the lowest part of the atmospheric surface layer.

1. Atmospheric diffusion: The extended spectrum model is relevant, for example, in the investigation of concentration fluctuations in smoke puffs and plumes released near the ground, where instantaneously released puffs of pollutants disperse on length scales covered by the shear production subrange. Here, diffusion of near-ground instantaneously released puffs have been found also to scale with the friction velocity, as does the shear production subrange, that is, $\sigma_{\text {Puff }} \propto u_{*} t$, where $\sigma_{\text {Puff }}$ is the standard deviation, or puff size, and $t$ the puff's diffusion time since release (cf., e.g., [33]).

2. Wind energy: For calculating loads and fatigues when turbine blade tips soars into the eddy surface layer near the ground, for instance at heights of 30-40 m and below, the standard 3D isotropic inertial subrange spectral models and simulations codes should possibly also be modified to take into account the different spectral form of the turbulence in the eddy surface layer.

3. Wind loads on bridges buildings: Similarly, spectral models and simulation codes used for load assessment and structural design of buildings and bridges could also be designed to take into account, where relevant to the height above the ground and the scale of turbulence considered, the added turbulence contained in the extended spectral model's shear production subrange.

Figure 6 shows only the spectra of the longitudinal wind component. However, the Høvsøre multiple height sonic anemometer data set also contains spectra of the transverse and vertical wind components.

Previously, [3] presented an eddy surface layer extended spectral model for the vertical wind component. A continuation of this study will therefore be to compare this extended vertical wind component spectrum model with vertical spectra at the six heights measured at $\mathrm{H} \varnothing \mathrm{v} \varnothing \varnothing \mathrm{re}$.

Similarly, the $H \varnothing v s \varnothing r e$ multiple height spectral data set also holds ensemble averaged spectra of the transverse wind component; it would therefore be possible to compare also the transverse wind component spectra with an extended spectra model similar to Eq. (3.3) for the transverse wind component.

\section{Conclusion}

An analysis of atmospheric surface layer spectra show that turbulence generated during near-neutral atmospheric stability conditions by strong shear within the lowest one-third of the surface layer, i.e., in the so-called eddy surface layer, follows a spectral form characteristic of a shear production subrange, $\sim u_{*}^{2} k^{-1}$ , as predicted previously by C. M. Tchen in 1953 - $1954[5,6]$.

A shear production subrange was added to the classical Kaimal spectrum model for neutral stratification, producing an extended spectral model for scaling and modeling the longitudinal wind component spectra on scales at which the eddy wavelength is larger than the observation or measurement height $h$ above the ground, i.e., for wavenumbers with wavelength $\lambda>h$. 
Similar to the classical Kaimal spectrum model, the shear production subrange extended spectral model is parameterized solely by the surface friction velocity, $u_{*}$, and the measurement height, $Z$ [cf. Eq. (3.3)].

The extended spectrum model appears to be able to scale the longitudinal spectra observed at Høvsøre better than the classical Kaimal spectrum model within the lowest one-third of the surface layer, corresponding to heights of up to $20-40 \mathrm{~m}$ above the ground.

\section{References:}

[1] Peña A, Floors R, Sathe A, Gryning S E, Wagner R, Courtney M S, Larsén X G, Hahmann A N and Hasager C B 2016 Ten Years of Boundary-Layer and Wind-Power Meteorology at Høvsøre, Denmark Boundary-Layer Meteorol. 158 1-26

[2] Hunt J C R and Carlotti P 2001 Statistical structure of the high Reynolds number turbulent boundary layers Flow, Turbul. Combust. 66 453-75

[3] Högström U, Hunt J C R and Smedman A-S 2002 Theory And Measurements For Turbulence Spectra And Variances In The Atmospheric Neutral Surface Layer Boundary-Layer Meteorol. 103 101-24

[4] Tchen C M, Larsen S E, Pécseli H and Mikkelsen T 1985 Large-Scale Spectral Structure with a Gap in the Stably Stratified Atmosphere Phys. Scr. 31 616-20

[5] Tchen C M 1953 On the Spectrum of Energy in Turbulent Shear Flow J. Res. Nat. Bur. Stand. 50 5162

[6] Tchen C M 1954 Transport Processes as Foundations of the Heisenberg and Obukhoff Theories of Turbulence Phys. Rev. 93 4-14

[7] Larsén X G, Vincent C and Larsen S E 2013 Spectral structure of mesoscale winds over the water $Q$. J. R. Meteorol. Soc. $139685-700$

[8] Larsén X G, Larsen S E, Petersen E L, Larsen X G, Larsen S E and Petersen E L 2016 Full-Scale Spectrum of Boundary-Layer Winds Boundary-Layer Meteorol. 159 349-71

[9] Larsen S E, Courtney M and Mahrt L 1990 Low frequency behaviour of horizontal velocity spectra in stable surface layers 9.th Symposium of Turbulence and Diffusion ed Larsen SE, K L and N O Jensen (Americam Meteorological Spciety) p 14.6

[10] Højstrup J 1982 Velocity Spectra in the Unstable Planetary Boundary Layer J. Atmos. Sci. 39 2239-48

[11] Olesen H R, Larsen S E and Højstrup J 1984 Modelling velocity spectra in the lower part of the planetary boundary layer Boundary-Layer Meteorol. 29 285-312

[12] Højstrup J, Larsen S and Madsen P 1990 Power spectra of horizontal wind components in the neutral atmospheric surface boundary layer 9. Symposium on turbulence and diffusion AMS Boston ed N Jensen, L Kristensen and S Larsen (Roskilde: AMS Boston) pp 305-8

[13] Monin A S and Yaglom A M . 1975 Statistical Fluid Mechanics: Mechanics of Turbulence ed J L Lumley (M.I.T. Press, Cambridge, Mass) 
[14] Kolmogorov A N 1941 The local structure of turbulence in incompressible viscous fluid for very large Reynolds numberst Compt. rend. acad. sci. U.R.S.S. 30301

[15] Obukhov A M 1941 On the distribution of energy in the spectrum of turbulent flow Dokl. Akad. Nauk SSSR p Vol 32, nos. 1, NaN-24

[16] Heisenberg W 1948 Zur statistischen Theorie der Turbulenz Z. Phys. 124 628-54

[17] von Kármán T 1948 Progress in the statistical theory of turbulence Proc. Natl Acad. Sci. pp 530-9

[18] Kaimal J C, Wyngaard J C, Izumi Y and Coté O R 1972 Spectral Characteristics of Surface-Layer Turbulence Q. J. R. Meteorol. Soc. 98 563-89

[19] Mann J 1998 Wind field simulation Probabilistic Eng. Mech. 13 269-82

[20] Kader B A, Yaglom A M and Zubkovskii S L 1989 Spatial correlation functions of surface-layer atmospheric turbulence in neutral stratification Boundary-Layer Meteorol. 47 233-49

[21] Hunt J C R and Morrison J F 2000 Eddy structure in turbulent boundary layers Eur. J. Mech. B-Fluids $19673-94$

[22] Perry a. E and Abell C J 1977 Asymptotic similarity of turbulence structures in smooth- and roughwalled pipes J. Fluid Mech. 79785

[23] Perry A E, Henbest S and Chong M S 1986 A theoretical and experimental study of wall turbulence J. Fluid Mech. 165 163-199

[24] Perry A E and Abell C J 1975 Scaling laws for pipe-flow turbulence J. Fluid Mech. 67257

[25] Perry A E and Marusic I 1995 A wall-wake model for the turbulence structure of boundary layers. Part 1. Extension of the attached eddy hypothesistle J. Fluid Mech. 298 361-88

[26] Townsend A A 1976 The Structure of Turbulent Shear Flow (Cambridge UK: Cambridge Unicersity Press)

[27] Gage K S and Nastrom G D 1986 Theoretical Interpretation of Atmospheric Wavenumber Spectra of Wind and Temperature Observed by Commercial Aircraft During GASP J. Atmos. Sci. 43 729-40

[28] Mikkelsen T, Ejsing Jørgensen H and Pécseli H L L 2001 Evidence in support of a k-1 Production subrange" in the near-neutral atmospheric surface layer 26th EGS General Assembly, Nice, France, 25/03/2001 - 30/03/2001, ed E G Society (Nice) p OA13

[29] Ejsing Jørgensen H, Astrup P, Mann J and Mikkelsen T 2006 Scaling of measured velocity spectra from the 168 meter tall met-tower at $\mathrm{H} \varnothing \mathrm{v} \varnothing \varnothing \mathrm{re}$, Denmark: Evidence of combined inertial K-5/3 and production K-1 subranges in the near-neutral atmospheric surface layer Proceedings EWEC 2006 (Brussels: European Wind Energy Association) p 1

[30] Mikkelsen T 1983 The Borris Field Experiment: Observations of Smoke Diffusion in the Surface Layer over Homogeneous Terrain Risø-R-479 (Technical University of Denmark DTU)

[31] Mikkelsen T and Eckman R 1985 Instantaneous Observations of Plume Dispersion in the Surface Layer Air Pollution Modeling and Its Application IV Volume 7 of the series Nato - Challenges of Modern Society ed C. de Wispelaere (Copenhagen: Plenum Press, New york 1985) pp 549-69 
[32] Jiang D 1985 On the Dispersion of Mixtures J. Fluid Mech. 155 309-26

[33] Mikkelsen T, Jørgensen H E, Nielsen M and Ott S 2002 Similarity scaling of surface-released smoke plumes Boundary-Layer Meteorol. 105 483-505 


\section{Nomenclature}

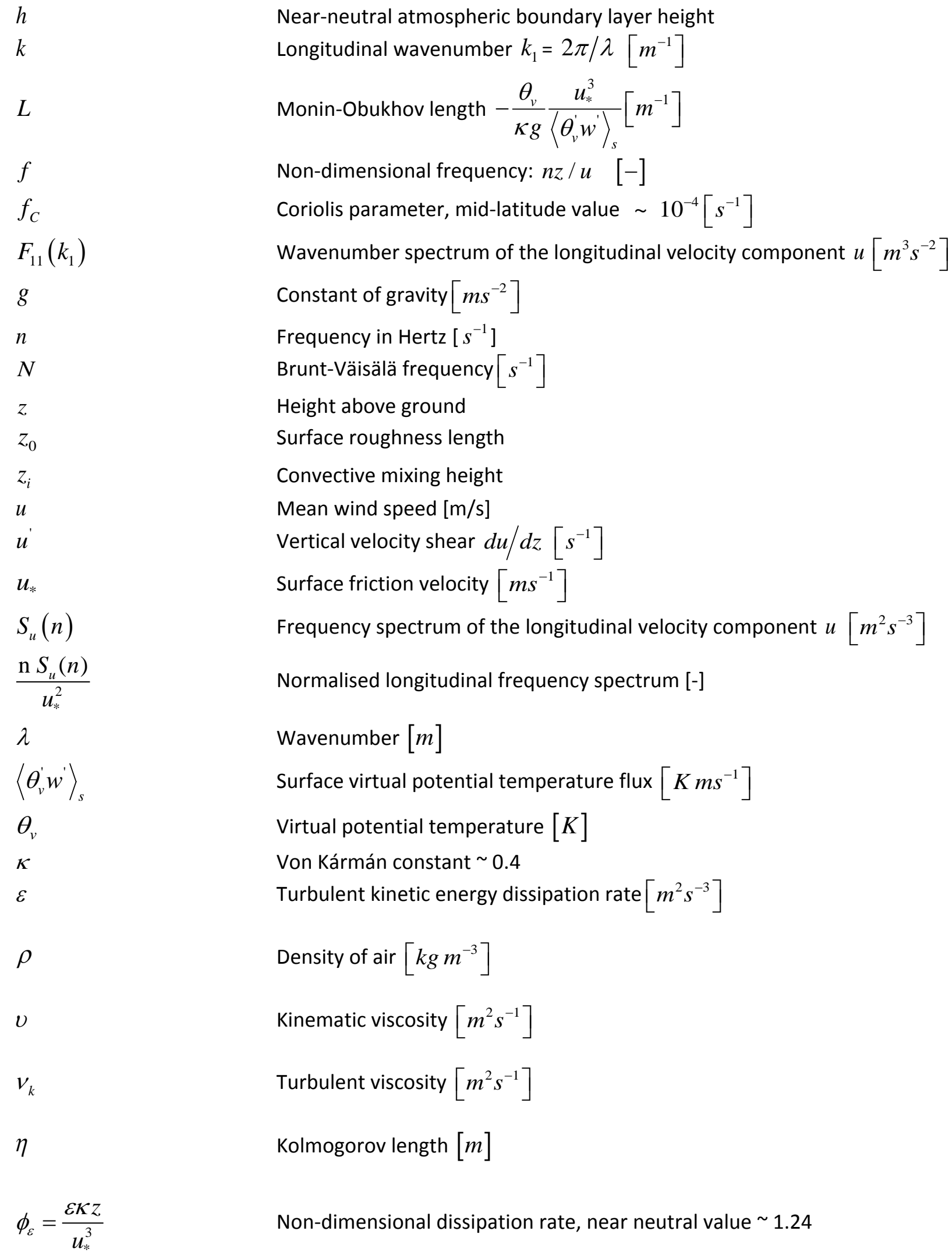

